\title{
FRIEND SUGGESTION SYSTEM FOR THE SOCIAL NETWORK BASED ON USER BEHAVIOR
}

\author{
Shamsu Shehu \\ Department of Mathematical Sciences, Al-Qalam University Katsina, Nigeria
}

\begin{abstract}
Now-a-days online social networks such as Facebook, Twitter, Google+, LinkedIn, and others have become significantly popular all over the world and people are using it throughout their daily lives. The number of users in the social networks is increasing day by day. Besides traditional desktop PCs and laptops, new emerging mobile devices makes it easier to make social networking. In online social network user behavior means various social activities that users can do online, such as friendship creation, content publishing, profile browsing, messaging, and commenting, liking, sharing and so on. So we are proposing to suggest one person to another person as a friend based these behaviors.
\end{abstract}

\section{KEYWORDS}

algorithm, graph mining, behaviors, social network, services

\section{INTRODUCTION}

Social Networking Sites (SNS) are the dominating entities in the modern web [2]Online friendship is now similarly appealing to people as offline friendship if not more. People tend to enjoy the fellowship of their real life friends in a virtual world. At the same time they are interested in making online friends. But With the rapid growth of SNS's resulting in information overload people are in dilemmas to choose the right friend and rightly so. The recent surge of research in recommendation algorithms is therefore not surprising. Popular movie recommendation site had even conducted competition with $\$ 1 \mathrm{M}$ grand prize for a $10 \%$ improvement on their movie recommendation algorithm [1]. However, recommendation of products and recommendation of human beings areas disparate as can be. Historically there has been two main recommendation algorithms content based and collaborative algorithm. Content based algorithm requires textual information as its name suggests and recommends websites newspaper articles and other contents. Collaborative based algorithm recommends products to a user which it believes have been light by similar users. Both of these algorithms have yielded unsatisfactory results in friend recommendation because of their lack of intuition in judging rational beings. We also try to improve the recommendation system on the basis of cohesiveness of friendship 


\section{LITERATURE REVIEW}

With the advent of Web 2.0, social computing has emerged as one of the hot research topics recently. In online social networks friends are recommended in various ways. But their solutions are not revealed yet. They may use different types of technique based on the activities of their users. So, for recommending friend a lot research is going on and a lot of research has been done. For understanding different behavior in the social network different approach may be taken. Connectivity and interaction, traffic activity, malicious behavior, mobile social behavior are needed for understanding user behavior in social network[1]. Some people use the photo comment and wall post as interaction to determine the behavior [2]. If the users are denoted as node and the friendship between different users are denoted as edge we can make a graph for recommending the friends. This graph may be formed as a directed graph or an undirected graph. There may be different types of graphs for the social graph, friendship graph where friendship between users are the edges, interaction graph where visible interaction, such as posting on a wall are the edges, latent graph Latent interaction, such as browsing profile are the edges, following graph subscribe to receive all messages are the edges [1]. Cluster analysis performed on the feature vectors may be used for identifying the user behaviors. From these cluster analysis dominant user behavior may be found using a threshold value [3]. If we can identify the user behaviors we can manage to suggest one person to another person to be his friend. By leveraging interest-based features, we can design a general friend recommendation framework by which we can recommend one person to be friend of another person [4]. Characterizing the user interest by two dimension, content and context we can build these framework [4]. Friend recommendation can be done using the clustered method [5]. Using the clustered method based on some factors the friends can be recommended. Twelve factors are used for defining the behavior. Among a lot of users first of all from this twelve features users are divided in five different clusters. This five clusters are formed by using K-means algorithm. Now if we select a focused user and plot it into the five clusters, and this user will match any of the five clusters. Then we can recommended this user into the users of that cluster.A cohesion based friend recommendation system are used for the friend recommendation system[6]. Cohesion based friend recommendation can be recommended by using some steps, extracting sub network from the whole network. Then measuring the link strength among the networks. After that a threshold value can be introduced for augmenting the network. Then we can recommend friends using community detection. Friend recommendation is based on the user social relations and personal information profiles or the "friend of friend" method [7]. Friends in common, similar age, geographic location are used to recommend friends. Users from same age same geographic location can be friends. User profiling system can be used for recommending friends [8]. Interpolation of profile data by the data of other users who are linked with the focused user. Friends are recommended based on clustering method [9]. Users browsing records and the content users interested in. Used clustering method for reducing system overhead while dealing with a large amount of data. It will make the friend recommendation much faster.

\section{Motivation}

The motivation behind our work is to recommend friends from the uncommon behavior. Here friends are not only recommended based on their uncommon behavior, but also first the common behaviors are be a part of recommendation. If we recommend friends only based on their common behavior, it will not be effective. Suppose one may like rock songs. So people who like 
rock songs should be friends. But this thing doesn't happen. Rock songs may be a common behavior but they may come from different communities, they works in different places, their other activities may not be the same. So if we use some common behavior as the parameter and based on that parameter if we find the common behaviors among those uncommon behaviors we can recommend friend very effectively.

\section{PROBLEM STATEMENT}

There are so many users in the social network. We cannot be able to recommend every one of them as friend. There should be some criteria to recommend one a friend. We want to propose a friend recommendation system based on user behavior for the social network. We will define our behavior first. Behavior will be divided into common and uncommon behavior. First common behavior will be defined. Then uncommon behavior will be defined Friends will be recommended from the uncommon behavior through the common behavior.

\section{METHODOLOGY}

Now-a-days, social networking sites like Facebook, Myspace, LinkedIn, twitter, Orkut etc. are very much popular to the people. Among of them Facebook is our sample social networking site for friend recommendation. The information of Facebook users will be used in our research. Behaviour means different activities. In Social Networking User Behaviour means different activities of the user in social network.

$\mathrm{U}=\{\mathrm{u} \mid$ users in Facebook $\}$

$\mathrm{A}=\{\mathrm{a} \mid$ activities of the users in Facebook $\}$

$\mathrm{R}=\mathrm{P}(\mathrm{A})$

So that,

$\mathrm{U}=\{\mathrm{u} 1, \mathrm{u} 2, \mathrm{u} 3, \ldots . . \mathrm{un}\}$

$\mathrm{A}=\{\mathrm{a} 1, \mathrm{a} 2, \mathrm{a} 3, \ldots \ldots, \mathrm{an}\}$

$\mathrm{R}=\{\{\mathrm{a} 1\},\{\mathrm{a} 2\},\{\mathrm{a} 3\}, \ldots \ldots,\{\mathrm{an}\},\{\mathrm{a} 1, \mathrm{a} 2\},\{\mathrm{a} 1, \mathrm{a} 3\}, \ldots . .,\{\mathrm{a} 1, \mathrm{a} 2, \mathrm{a} 3, \ldots \ldots, \mathrm{an}\}\}$

Now the behavior of the user will be formulated by a function $B$,

$\mathrm{B}: \mathrm{U} \rightarrow \mathrm{R}$

So, $B=\{b 1, b 2, b 3, \ldots . ., b n\}$

So that,

$\mathrm{b} 1$ = behavior of the $\mathrm{u} 1$

$\mathrm{b} 2$ = behavior of the $\mathrm{u} 2$

$\mathrm{b} 3$ = behavior of the $\mathrm{u} 3$

•

$\mathrm{bn}=$ behavior of the un

Now the behavior of the users is depending on the activities of the users.

So, $\mathbf{B}$ is a proper subset of $\mathbf{R}$.

Now ,B is the relation of the activities.

This relation can be defined as many formulation as it is defined in the discussion. 
Suppose user $\mathrm{u} 1$ has the activity a1, a2, a3, ..., an

And every activity is independent then

$\mathrm{b} 1=\{\mathrm{a} 1, \mathrm{a} 2, \mathrm{a} 3, \ldots . ., \mathrm{an}\}$

If a1, a2, a3, ....., an are dependent to each other then the user behavior can be represented as a complex graph where activities will be the node and relation of the activities as edges of the graph G, then
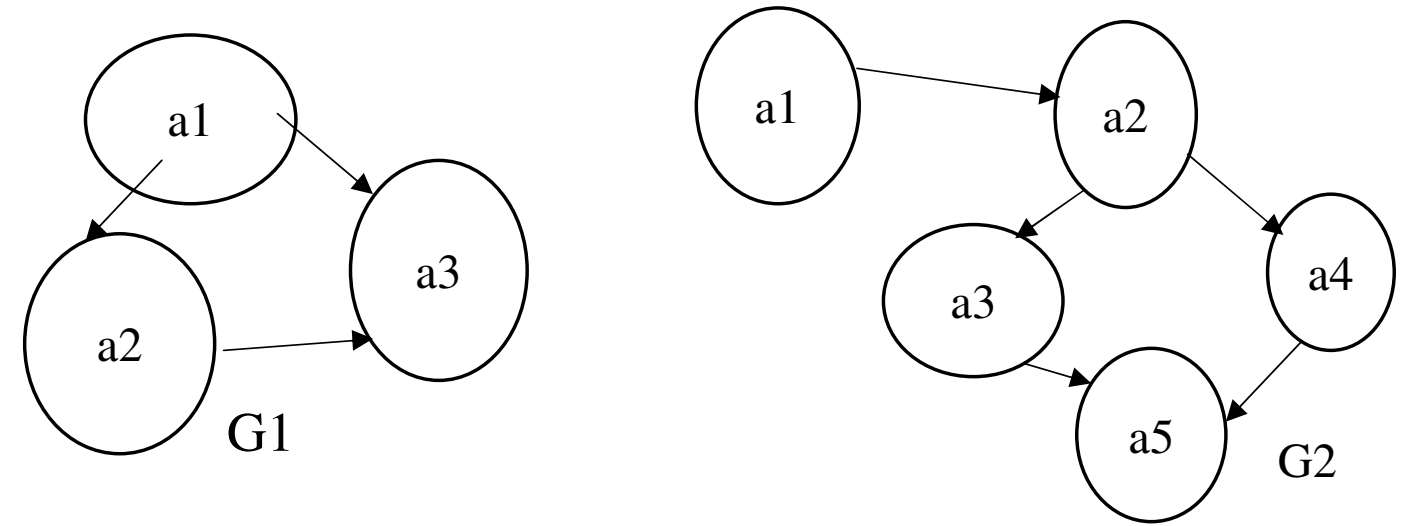

So, $\mathrm{b} 1=\{\mathrm{G} 1, \mathrm{G} 2, \mathrm{G} 3, \ldots \ldots, \mathrm{Gn}\}$

Again sequence of the activity can also be the behavior of the user

$\mathrm{S} 1=\mathrm{a} 1 \rightarrow \mathrm{a} 2 \rightarrow \mathrm{a} 3 \rightarrow \mathrm{a} 5 \rightarrow \mathrm{a} 6 \rightarrow \mathrm{a} 3 \rightarrow \mathrm{a} 4$

$\mathrm{S} 2=\mathrm{a} 1 \rightarrow \mathrm{a} 2 \rightarrow \mathrm{a} 5 \rightarrow \mathrm{a} 7 \rightarrow \mathrm{a} 8$

These sequence of the activities $\mathrm{S} 1, \mathrm{~S} 2, \mathrm{~S} 3, \ldots ., \mathrm{Sn}$ can be the behavior of the user $\mathrm{u} 1$.

So, $b 1=\{\mathrm{S} 1, \mathrm{~S} 2, \mathrm{~S} 3, \ldots, \mathrm{Sn}\}$

Common Behaviour:

- Common behaviour means the common activities of the user

- In social networking it is not fixed

- Differs from domain to domain

Uncommon Behaviour:

- Uncommon behaviour means the uncommon activities of the user

- Like common behaviour, it is also not fixed

- Differs from domain to domain

This two terms can vary by the values of the behaviour analysis. These two behaviour can be determined by the mining.If graph is used then graph mining will be used as mining.If sequence then sequence mining will be used.It can be differentiate by a threshold value,

$\mathrm{H}$

If $\mathrm{B}(\mathrm{u})>\mathrm{H}$, it is common

If $\mathrm{B}(\mathrm{u})<=\mathrm{H}$, it is uncommon

We will use this factors:

- Comment, like, photo comment

- Similar group

- School, town, job, interest

- Community

- Daily Activities 
International Journal of Computer Science, Engineering and Information Technology (IJCSEIT), Vol.7, No.5, October 2017

\section{CONCLUSION}

We have found this research area very interesting to us. This research helps in recommending friends in online social networks very effectively. There may be some limitations that we will have to work with a lot of parameters and we have to determine which will be the common behavior and which will be the uncommon behavior. This is the most challenging task for recommending friends. So we are trying to find a way to recommend friend most effectively.

\section{REFERENCES}

[1] LongJin et al (2013), "Understanding User Behavior in Online Social Networks: A Survey”

[2] C. Wilson et al (2009), "User Interactions in Social Networks and Their Implications,"

[3] Marcelo Maia et al, "Identifying User Behavior in Online Social Networks"

[4] Xing Xie (2010), "Potential Friend Recommendation in Online Social Network"

[5] Francis T. O'Donovan et al (2013), “Characterizing user behavior and information propagation on a social multimedia network"

[6] Md. Nafiz Hamid (2014), “A cohesion-based friend-recommendation system”

[7] MichealMoricz et al (2010), "PYMK: friend recommendation at myspace"

[8] Hajime Hotta et al (2007), "User Profiling System Using Social Networks for Recommendation"

[9] Zhiwei Deng et al (2012), "Personalized friend recommendation in social network based on clustering method" 